\section{$\underset{\substack{\text { hommes } \\ \text { \& migrations }}}{ }$}

\section{Hommes \& migrations}

Revue française de référence sur les dynamiques

migratoires

$1296 \mid 2012$

Le Mexique dans les migrations internationales

\title{
Migration et développement
}

De l'ambivalence à la désillusion?

Silvia E. Giorguli Saucedo et Edith Y. Gutiérrez

Traducteur : Bernardo Toro

\section{(2) OpenEdition}

\section{Journals}

\section{Édition électronique}

URL : http://journals.openedition.org/hommesmigrations/1703

DOI : 10.4000/hommesmigrations.1703

ISSN : 2262-3353

\section{Éditeur}

Musée national de l'histoire de l'immigration

\section{Édition imprimée}

Date de publication : 1 mars 2012

Pagination : 22-33

ISSN : 1142-852X

Référence électronique

Silvia E. Giorguli Saucedo et Edith Y. Gutiérrez, « Migration et développement », Hommes \& migrations [En ligne], 1296 | 2012, mis en ligne le 31 décembre 2014, consulté le 01 mai 2019. URL : http:// journals.openedition.org/hommesmigrations/1703; DOI : 10.4000/hommesmigrations.1703 


\section{Migration et développement De l’ambivalence à la désillusion?}

Par Silvia E. Giorguli Saucedo, professeur et directrice du Centre d'études démographiques, urbaines et environnementales, El Colegio de Mexico et Edith Y. Gutiérrez, Centre d'études démographiques, urbaines et environnementales, El Colegio de Mexico

Dans un contexte d'incertitude économique et politique, la migration vers les États-Unis est de moins en moins envisagée par les Mexicains comme une opportunité, phénomène qui se combine

à un important flux de migrants de retour. Pour la première fois, le solde migratoire est proche de zéro. Ce nouveau scénario permet de réévaluer les modestes contributions de la migration internationale

au développement local et de repenser l'impact de la migration, tout comme les défis que ces changements impliquent pour l'avenir. 
L'histoire de la migration entre le Mexique et les États-Unis a été marquée par des moments de basculement qui ont généré des paramètres, des tendances et des acteurs nouveaux ${ }^{(1)}$. Parmi ces changements, celui qui est intervenu au cours de la deuxième moitié des années quatre-vingt est le résultat de multiples facteurs : la croissance exceptionnelle du flux migratoire, la rupture de la circularité du flux, la formation d'une communauté mexicaine aux États-Unis qui compte aujourd'hui près de douze millions de personnes ${ }^{(2)}$, l'expansion du phénomène migratoire sur l'ensemble du territoire mexicain (qui s'oppose à la concentration dans certaines régions) et l'augmentation des envois de fonds aux foyers et communautés d'origine. Ces aspects résument la tendance de la mobilité entre le Mexique et les États-Unis au cours des trois dernières décennies, depuis le milieu des années quatre-vingt jusqu'au milieu des années deux mille.

Les données récentes signalent un changement important dans les tendances de la migration entre le Mexique et les États-Unis ${ }^{(3)}$. La diminution considérable du nombre de Mexicains qui partent pour les États-Unis, l'augmentation du nombre de retours au Mexique et le solde migratoire net proche de zéro résument ce changement. Au-delà de la conjoncture économique et politique aux États-Unis, nous avons affaire à une nouvelle étape dans l'histoire de l'émigration mexicaine. Devant cet état de choses, deux réflexions s'imposent. La première nous amène à reconsidérer l'impact de la migration mexicaine aux États-Unis sur le développement local. La deuxième nous invite à repenser la relation entre migration et développement local, à partir des nouveaux modèles de mobilité entre les deux pays et face aux discussions théoriques prédominantes sur les liens entre migration internationale et développement.

\section{Rapport entre migration et développement local : le cas mexicain}

Le débat théorique sur l'effet potentiel de la migration internationale sur le développement local a oscillé entre des visions optimistes et pessimistes ${ }^{(4)}$. Vers la fin des années soixante et au moins jusqu'au début des années quatre-vingt, la migration était perçue comme l'expression de la dépendance des économies moins développées, elle contribuait, pensait-on, à la reproduction d'un modèle d'inégalité et de stagnation $^{(5)}$.Suivant cette perspective, fondée sur une vision théorique structuraliste, on estimait que les bénéfices économiques de la migration internationale pour les communautés d'origine étaient nuls ou marginaux.

Au Mexique, cette vision coïncide avec l'étape de croissance de la migration clandestine, après la période Bracero ${ }^{(6)}$. Durant cette période, la migration internationale gardait 
un caractère rural, circulaire et était fondamentalement masculine. Elle n'avait pas encore pris l'importance qu'elle a atteinte par la suite et n'était pas censée favoriser le développement local.

Au cours de la dernière décennie du vingtième siècle, il s'est opéré un changement dans la perception du rapport entre migration et développement. La constitution de communautés de migrants à l'étranger qui envoient de l'argent à leur pays d'origine et l'augmentation de ces envois a provoqué un regain d'optimisme sur le rôle possible de la migration et des migrants dans le développement local ${ }^{(7)}$. Dans ce contexte, la discussion sur le bénéfice potentiel des transferts de fonds aux foyers a pris de l'ampleur. On s'est aperçu que cet apport d'argent générait une croissance de la consommation locale et pouvait se transformer en investissement productif. De la même manière, on a commencé à développer des arguments sur les contributions des diasporas migrantes au développement local à travers le transfert de connaissances et de ressources (infrastructures, projets productifs, etc.). Ce changement de vision sur le rapport entre migration et développement a conféré aux migrants le rôle d'acteurs principaux du processus, de véritables "héros du développement"(8).

Au Mexique cette discussion a coïncidé avec l'augmentation sans précédent de la migration vers les États-Unis. La population mexicaine aux États-Unis est passée de 2,2 millions en 1980 à environ 5 millions en 1990, puis à 9,5 millions autour de l'année 2000. En 2010, elle s'est stabilisée autour de 12,5 millions ${ }^{(9)}$. Cette année, $10 \%$ de la population née au Mexique vivait aux États-Unis. Parallèlement, les envois de fonds passent de 6,5 milliards de dollars en 2000 à presque 25 milliards en $2007^{(10)}$. Durant cette période, la perception de la migration change. On réalise diverses études pour évaluer l'impact dans les économies locales des envois de fonds des migrants en vue de financer des projets productifs et d'infrastructures. Au niveau gouvernemental, on développe diverses actions autour de l'accueil de ressources liées aux remises de fonds ou bien à travers les organisations de migrants ; parmi ces actions citons les programmes $3 \times 1$ qui complètent l'apport des migrants par les investissements des États et du gouvernement fédéral.

\section{Des envois de fonds à géométrie variable}

Après une décennie de politiques nationales ou de programmes financés par des organisations internationales destinées à faire fructifier les bénéfices des envois de fonds des migrants, les résultats sont peu concluants et provisoires ${ }^{(11)}$. On trouve des exemples positifs dans divers pays d'origine, cependant leur portée est limitée et les expériences restent très hétérogènes. Certaines études, beaucoup d'entre elles sur le 
Mexique, montrent même le contraire. Suivant une perspective économique, sociologique ou anthropologique, elles pointent les effets négatifs de la migration internationale sur les communautés d'origine ${ }^{(12)}$. Après l'optimisme excessif sur les bienfaits des envois de fonds, des doutes ont surgi sur le rôle des migrants comme promoteurs du développement. On signale que lesdits envois sont des transferts privés qui ne sont pas destinés à être investis dans le développement, les politiques migratoires dans les pays d'origine ne pouvant pas se substituer aux politiques de développement social plus profondes.

Même si le débat n'est pas clos, l'absence de résultats probants a créé un certain

Après l'optimisme
excessif sur les bienfaits
des envois de fonds,
des doutes ont surgi
sur le rôle des migrants
comme promoteurs
du développement.
consensus autour de l'idée que les envois de fonds ne peuvent pas constituer à eux seuls une source de développement ${ }^{(13)}$. Aussi bien au Mexique que dans bien d'autres pays, on estime que la migration internationale interagit avec d'autres processus sociaux et économiques plus vastes. Son impact sur les communautés d'origine dépend aussi des contextes institutionnels et des normes sociales où elle se développe.

Une discussion parallèle porte sur le profil du flux migratoire et son lien avec les processus de développement local. La formation de diasporas dans les pays d'accueil qui s'organisent en associations et envoient des ressources aux communautés d'origine est le résultat d'un changement au sein de ces diasporas, lesquelles se sont sédentarisées et parfois ont fait venir le reste de la famille. L'allongement de la durée du séjour dans le pays d'accueil, la stabilité et l'amélioration de la situation économique permettent le flux permanent des ressources monétaires sous la forme de remises de fonds familiales ou de transferts d'argent à travers les organisations de migrants. Néanmoins, l'absence de circularité des flux migratoires génère, par ailleurs, un phénomène de dépeuplement ${ }^{(14)}$ et, éventuellement, conduit à une situation où le flux des envois de fonds tendrait à diminuer quand les familles complètes émigrent ou quand les enfants et les autres descendants ne gardent pas de liens avec leur lieu d'origine. En partant de ces arguments, certains soutiennent que pour avoir un impact positif sur les communautés d'origine, la migration internationale doit garder son caractère temporaire ${ }^{(15)}$.

Les envois de fonds restent une source importante de richesse pour les foyers et les pays qui les reçoivent, les arguments sur leur évolution sont avant tout des hypothèses sur l'avenir. Toutefois la crise économique récente aux États-Unis et les fluctuations dans l'envoi de fonds lors des trois dernières années laissent voir que les montants 
desdits envois dépendent des conjonctures économiques. Cela constitue une raison supplémentaire pour mettre en question les envois de fonds dans la stratégie de développement global.

\section{Nouveaux profils migratoires et développement local}

Le climat restrictif et criminalisant qui pèse sur la migration illégale aux États-Unis, la crise économique et l'augmentation du chômage dans les secteurs de plus forte concentration de Mexicains a entraîné un changement dans les flux migratoires ces trois dernières années. Il est à signaler que depuis le milieu des années deux mille, on observe un ralentissement de la croissance de la population mexicaine installée aux États-Unis et une diminution du flux d'entrées ${ }^{(16)}$. Entre 2007 et 2010 on a constaté une chute brutale du flux de sorties, la réduction de l'envoi de fonds et l'augmentation de la migration de retour ${ }^{(17)}$. Étant donné le rapport étroit entre les flux migratoires de Mexicains aux États-Unis et les variations du marché de l'emploi, on peut estimer que si l'économie américaine sort de la crise et que le taux d'emploi augmente le flux migratoire se réactivera et le retour des Mexicains à leur lieu d'origine diminuera. Il est possible toutefois que les niveaux connus par le passé ne soient pas atteints et que les flux se stabilisent autour de chiffres moins élevés que ceux de la première moitié de la dernière décennie.

Les changements récents dans les profils migratoires apportent de nouveaux éléments au débat autour de la migration et du développement. Deux éléments doivent retenir notre attention. D'un côté, le nombre d'émigrants a diminué considérablement. D'après les recensements de 2000 et 2010, entre 1995 et 2000, 1,5 million de personnes ont quitté le pays. Ce chiffre tombe à moins d'un million dix ans plus tard (tableau 1).D'un autre côté, on a cru que la crise économique américaine provoquerait un retour massif des migrants au Mexique. Les statistiques indiquent que le changement a été réel. Si dans la période 1995-2000 on a enregistré moins de 300000 retours, dans la période 2005-2010, le nombre de retours s'est chiffré à 800 000, auxquels il faut ajouter 356000 Mexicains nés aux États-Unis qui sont allés vivre au Mexique (tableau 1). Ces derniers sont pour la plupart âgés de moins de 15 ans (tableau 2), parmi lesquels plus de 300000 vivent dans des foyers dont le chef de famille est mexicain ${ }^{(18)}$. L'augmentation du nombre de retours de migrants coïncide avec la stabilisation du nombre de Mexicains aux États-Unis. D'après Passel ${ }^{(19)}$, la population résidant aux États-Unis et née au Mexique est restée stable au cours des trois dernières années. Cette donnée est importante car elle permet de comprendre 
Tableau 1 : Distribution de la population selon un statut social migratoire, Mexique

\begin{tabular}{lrrrrrr}
\hline \multirow{2}{*}{ Expériences migratoires } & \multicolumn{3}{c}{2000} & \multicolumn{3}{c}{2010} \\
\cline { 2 - 7 } & \multicolumn{1}{c}{ Absolus } & \multicolumn{1}{c}{ Relatif } & Absolus & \multicolumn{1}{c}{ Relatif } \\
\hline Non migrants & 95519551 & 96,9 & & 109455646 & 96,9 & \\
Migrants de retour & 267150 & 0,2 & 11,4 & 824436 & 0,7 & 29,7 \\
Immigrants E.U. récents & 171690 & 0,2 & 7,3 & 356695 & 0,3 & 12,8 \\
Autres immigrants E.U. & 184458 & 0,2 & 7,8 & 377197 & 0,3 & 13,6 \\
Autres immigrants récents & 44018 & 0,0 & 1,9 & 68511 & 0,1 & 2,5 \\
Autres immigrants & 116703 & 0,1 & 5,0 & 158773 & 0,1 & 5,7 \\
Migrants & 1569157 & 1,4 & 66,7 & 994869 & 0,9 & 35,8 \\
N.E. & 711297 & 0,6 & & 718881 & 0,6 & \\
Total & 98584024 & 100,0 & 100,0 & 112955008 & 100,0 & 100,0 \\
\hline
\end{tabular}

Note : La situation migratoire se définit à partir des changements de lieu de résidence entre 1995 et 2000 , et entre 2005 et 2010 , et du lieu de naissance.

Source : Estimations propres basées sur les données issues des recensements effectués en 2000 et 2010, Inegi.

Tableau 2 ; Quelques caractéristiques de la population selon sa situation migratoire en 2010

\begin{tabular}{|c|c|c|c|c|c|c|}
\hline Caractéristi & & Non migrants & $\begin{array}{l}\text { Migrants de } \\
\text { retour }\end{array}$ & $\begin{array}{l}\text { Immigrants } \\
\text { E.U. récents }\end{array}$ & $\begin{array}{c}\text { Autres } \\
\text { immigrants }\end{array}$ & Migrants \\
\hline Total & & $\begin{array}{r}109455646 \\
96,9\end{array}$ & $\begin{array}{r}824436 \\
0,7\end{array}$ & $\begin{array}{r}356695 \\
0,3\end{array}$ & $\begin{array}{r}68511 \\
0,1\end{array}$ & $\begin{array}{r}994869 \\
0,9\end{array}$ \\
\hline Sexe & Homme & 48,5 & 72,0 & 50,8 & 51,4 & 76,7 \\
\hline Âge & 0 a 14 & 29,0 & 5,8 & 86,5 & 20,6 & 3,6 \\
\hline Localité & Moins de 2500 hab & 23,5 & 34,7 & 25,6 & 11,1 & 42,0 \\
\hline & Pop & ation âgées de & 5 à 34 ans & & & \\
\hline Scolarité & $\begin{array}{l}\text { Médias Supérieurs } \\
\text { Supérieurs }\end{array}$ & $\begin{array}{l}19,7 \\
23,1\end{array}$ & $\begin{array}{r}20,3 \\
7,7\end{array}$ & $\begin{array}{l}41,8 \\
34,5\end{array}$ & $\begin{array}{l}13,4 \\
69,0\end{array}$ & - \\
\hline Secteur d'activité économique & $\begin{array}{l}\text { Primaire } \\
\text { Secondaire } \\
\text { Tertiaire }\end{array}$ & $\begin{array}{r}6,3 \\
17,6 \\
41,1\end{array}$ & $\begin{array}{l}17,3 \\
21,8 \\
31,3\end{array}$ & $\begin{array}{r}1,9 \\
9,7 \\
40,5\end{array}$ & $\begin{array}{r}2,1 \\
9,1 \\
48,6\end{array}$ & -- \\
\hline & Total & 16649012 & 309174 & 11924 & 17507 & --- \\
\hline
\end{tabular}

Note : La situation migratoire se définit à partir des changements de lieu de résidence entre 2005 et 2010 , et du lieu de naissance.

Source : Estimations propres basées sur les données issues du recensement effectué en 2010, Inegi.

que l'incidence de la hausse du taux de retours sur le nombre total de Mexicains résidant aux États-Unis est très faible.

Pour contextualiser les défis que le nouveau profil migratoire implique en termes de développement pour les communautés d'origine, il faut les mettre en rapport avec le profil des migrants (ceux qui sont partis et ceux qui sont retournés). Par ailleurs, ainsi qu'on peut le voir dans les cartes 1,2 et 3, la distribution de la migration, la chute des envois de fonds et le retour ont une composante régionale, ils varient le long du 
territoire mexicain et sont liés au niveau de consolidation de la migration dans les régions avec une plus ou moins grande tradition migratoire.

Le tableau 2 montre quelques caractéristiques de la population répartie selon son statut migratoire. On remarque qu'aussi bien chez les émigrants que chez les migrants de retour, le flux est principalement masculin (plus de $70 \%$ dans les deux cas) et plus fort dans les localités rurales. Alors que 23,5\% de la population non migrante habitait dans des localités de moins de 2500 habitants, cette proportion est plus forte entre les migrants de retour (34,7\%) et les émigrants (42\%). Une autre donnée qui retient notre attention est la différence de profil éducatif et de secteur d'activité. Les migrants de retour ont des niveaux de scolarité moins élevés que la population non migrante et participent davantage à des activités agricoles (secteur primaire) (tableau 2). En ce qui concerne la dimension géographique, la carte 1 montre clairement comment l'émigration diminue sur l'ensemble du territoire, même si le changement est plus important dans la région de plus grande tradition migratoire (ouest du pays). Chez les foyers qui reçoivent des versements de fonds le changement est moins marqué. Au niveau national, nos estimations indiquent que le pourcentage de foyers qui reçoivent des envois de fonds est passé de 4,5 à 3,6 entre 2000 et 2010, ce qui représente une baisse modérée. La diminution est plus importante dans la région traditionnelle (ouest de Mexique, voir carte 2) où le pourcentage est passé de 9,5 à 7,2 dans la même période. Finalement le retour des Mexicains apparaît aussi comme un phénomène qui s'étend sur tout le territoire national. Dans pratiquement toutes les régions migratoires la présence de migrants de retour augmente (carte 3), surtout dans les communes de la

Carte 1 : Proportion de foyers avec au moins un émigrant aux États-Unis par municipalité, Mexique 
Carte 2 : Proportion de foyers bénéficiaires de remises migratoires en 2000 et 2010

Source : Estimations propres basées sur les données issues des recensements effectués en 2000 et 2010, Inegi.

Source : Estimations propres basées sur les données issues des recensements effectués en 2000 et 2010, Inegi.

région traditionnelle et frontalière, ce qui pourrait indiquer une différence entre un flux de migrants qui retournent à leurs communautés d'origine et un autre qui s'installe près de la frontière avec l'idée probablement de retourner aux États-Unis si la situation économique et politique s'améliore. Pour les moins de 15 ans nés aux États-Unis qui vivaient au Mexique en 2010, cette population a aussi augmenté considérablement et, ainsi que nous l'avons dit, s'est surtout installée dans la région frontalière. Dans un nombre important de communes frontalières entre 10 et $20 \%$ des foyers comptaient au moins un enfant né aux États-Unis. 
Qu'est-ce que ces changements impliquent dans l'évolution du lien entre migration et développement au Mexique? Une première réflexion nous amène à reconsidérer le rôle joué par l'émigration internationale vers les États-Unis comme voie de secours face aux crises économiques récurrentes et le manque de perspectives de travail au Mexique durant les trois dernières décennies ${ }^{(20)}$. Devant les pressions économiques dues à la croissance de la population active dans un contexte économique défavorable, l'émigration a représenté une solution pour des millions de Mexicains. Aujourd'hui, environ 17 \% de la population active mexicaine réside aux États-Unis. Le changement de flux indique un éventuel épuisement de ce mécanisme, sans que l'on ait pour autant résolu les problèmes du marché de travail au Mexique. Cela peut s'avérer préoccupant pour "la stabilité économique, sociale et politique du pays ${ }^{(21)}$ ".

\section{La pression des flux de retours}

D'un autre côté, le retour des migrants, la plupart en âge de travailler, entraîne de nécessaires créations d'emplois dans les lieux d'origine. Ce retour coïncide avec une augmentation du taux de chômage jamais vue auparavant ${ }^{(22)}$. Ces deux changements intervenus dans la dynamique migratoire - moins de sorties et plus de retours accroissent la pression sur le marché de l'emploi national, notamment dans certaines régions. Il faut donc mettre en place des politiques de travail qui s'insèrent dans ce nouveau contexte de la dynamique migratoire et qui, étant donné les variations régionales du marché de l'emploi, tiennent compte des caractéristiques locales. Dans la discussion sur le lien entre migration et développement, on évoque constamment la possibilité de capitaliser le retour des migrants pour encourager le développement local. La mise à profit des connaissances et des compétences acquises à l'étranger et l'investissement de ressources que les migrants apportent avec eux peuvent favoriser la croissance économique et le bien-être des communautée ${ }^{(23)}$. Pour que cet apport puisse avoir lieu, il faut toutefois créer les conditions nécessaires dans les contextes d'origine. Dans les flux de retour, on rencontre un éventail d'expériences très hétérogènes. Il y a ceux qui retournent avec un capital et de nouvelles connaissances après un long séjour à l'étranger, ceux qui ont été renvoyés à la frontière et retournent dans une situation de grande vulnérabilité, ceux qui ont gardé des liens plus étroits avec leur communauté d'origine et peuvent de ce fait se réinsérer plus facilement, ceux enfin qui sont partis très jeunes et n'ont presque plus de liens avec leur communauté d'origine. Pour l'État mexicain et les gouvernements locaux, le défi consiste à générer les opportunités qui permettent de rentabiliser les bénéfices du retour, en tenant compte, bien entendu, du caractère hétérogène des situations.

Les mineurs qui sont entrés dans le pays, quelques-uns nés au Mexique, d'autres aux 
États-Unis, constituent un autre exemple des situations générées par l'augmentation de la migration de retour. Au cours des cinq dernières années, près de 380000 mineurs sont arrivés au Mexique ${ }^{(24)}$. Ainsi que nous l'avons avancé, dans certaines communes un nombre important de foyers a au moins un enfant dans cette situation. De nouvelles études sont nécessaires dans ce domaine pour comprendre les défis que le système éducatif mexicain devra assumer pour intégrer ces mineurs et mettre à profit les connaissances et ressources qu'ils apportent de leur expérience scolaire aux États-Unis. Mais la nouvelle dynamique migratoire génère aussi de nouvelles vulnérabilités. La fluctuation du montant et de la fréquence des envois de fonds aura surtout des effets

Pour l'État mexicain
et les gouvernements locaux,
le défi consiste à générer
les opportunités qui
permettent de rentabiliser
les bénéfices du retour,
en tenant compte,
bien entendu, du caractère
hétérogène des situations.
sur les foyers qui en dépendent financièrement et en particulier sur ceux dont ces versements constituent la seule source de revenus. En raison de la présence de mineurs ou de personnes âgées, les foyers qui bénéficient de ces transferts de fonds sont extrêmement dépendants. Pour faire face aux situations d'urgence économique, ils auront sans doute besoin d'un appui, si la fluctuation des envois de fonds persiste.

Le chômage et les politiques locales restrictives exposent les Mexicains installés aux États-Unis à une situation de plus grande vulnérabilité. Au cours de la dernière décennie, les écarts de salaire entre les Mexicains et les natifs n'ont cessé de se creuser, même les travailleurs mexicains installés depuis longtemps aux États-Unis en ont pâti ${ }^{(25)}$. Il est possible que cette situation s'aggrave encore dans les années qui viennent et qu'elle affecte aussi bien les migrants que leurs descendants.

\section{Conclusion}

Les changements récents intervenus dans la dynamique des flux entre le Mexique et les États-Unis apportent de nouvelles pistes de réflexion au débat actuel sur la migration internationale et le développement. D'une part, ils mettent en question la perception des envois de fonds comme facteur favorisant le développement local. Les fluctuations desdits envois et le scénario économique défavorable aux États-Unis indiquent, en effet, qu'il n'est pas possible de compter sur ces transferts pour financer l'infrastructure sociale et l'investissement économique dans les communautés d'origine. D'autre part, dans le débat sur les conditions nécessaires pour favoriser le développement local on parle de la nécessité de préserver la circularité des flux et de 
promouvoir le retour ${ }^{(26)}$. Dans cette perspective, le retour d'un nombre important de Mexicains pourrait être envisagé comme une chance. Pour qu'elle devienne une réalité, il faudra toutefois que les conditions soient offertes aux migrants de développer leurs connaissances, compétences et ressources, notamment dans le domaine de l'emploi. Une nouvelle attitude visant à dissuader les Mexicains de migrer vers les États-Unis constitue un autre changement important ${ }^{(27)}$. L'évaluation des pertes provoquées par la diminution de la population active joue un grand rôle dans cette nouvelle attitude. Si l'on parvient à générer un consensus autour de cette question, il faudra mettre en place des programmes spécifiques qui génèrent des possibilités d'emploi au niveau national, notamment en direction de la population jeune et des régions à fort taux d'émigration.

\section{Traduit de l'espagnol par Bernardo Toro}

\section{Notes}

1. Jorge Durand et Douglas Massey, Clandestinos. Migración México-Estados Unidos en los albores del siglo XXI, México, Miguel Ángel Porrúa, 2003.

2. Jeffrey S. Passel, "Flujos migratorios México-Estados Unidos de 1990 a 2010: Un análisis preliminar basado en las fuentes de información estadounidenses", in Coyuntura Demográfica, n ${ }^{\circ}$, 2011, pp. 15-20.

3. Jeffrey S. Passel et D'Vera Cohn, Unauthorized Immigrant Population: National and State Trends, 2010, Washington, Pew Hispanic Center, 2011. http://pewhispanic.org/reports/report.php? ReportID=133 ; Jeffrey S. Passel, "Flujos migratorios México-Estados Unidos de 1990 a 2010: Un análisis preliminar basado en las fuentes de información estadounidenses", op. cit.; René Zenteno, "Recent trends in mexican migration to US: the Mexico perspective", exposé présenté au 2011 Annual Meeting de la Population Association of America, Washington, 2 avril 2011.

http://www.somede.org/documentos/zenteno-2011.pdf.

4. Francisco Alba, "Migración internacional y políticas públicas", in Paula Leite et Silvia Giorguli (dir.), El estado de la migración. Las políticas públicas ante los retos de la migración mexicana a Estados Unidos, México, Consejo Nacional de la Población, 2009, pp. 23-46 ; Alejandro Portes, "Migración y desarrollo: una revisión conceptual de la evidencia", in Stephen Castles et Raúl Delgado Wise (dir.), Migración y desarrollo: perspectivas desde el sur, México, université autonome de Zacatecas, Institut national de migration et Miguel Ángel Porrúa, 2007 ; Hein De Haas, "Migration and development. A theoretical perspective", in Cahier de travail, $\mathrm{n}^{\circ}$ 9, International Migration Institute, Oxford University, 2008 ; Stephen Castles, "Development and migration - Migration and development: What comes first ?", exposé présenté à la Social Science Research Council Conference, Migration and Development: Future Directions for Research and Policy, février 2008, New York.

5. Hein De Haas, "Migration and development. A theoretical perspective", op. cit.; Stephen Castles, "Development and migration - Migration and development: What comes first?", op. cit.

6. Jorge Durand et Douglas Massey, Clandestinos. Migración México-Estados Unidos en los albores del siglo XXI, op. cit.

7. Hein De Haas, "Migration and development. A theoretical perspective", op. cit.

8. Stephen Castles, "Development and migration - Migration and development: What comes first?", op.cit., p. 3.

9. Jeffrey S. Passel, "Flujos migratorios México-Estados Unidos de 1990 a 2010: Un análisis preliminar basado

en las fuentes de información estadounidenses", op. cit.

10. Francisco Alba, "Migración internacional y políticas públicas", op. cit., p. 37.

11. Hein De Haas, "Migration and development. A theoretical perspective", op. cit.

12. Ibid.

13. Francisco Alba, "Migración internacional y políticas públicas", op. cit.; Alejandro Portes, "Migración y desarrollo: una revisión conceptual de la evidencia", op. cit.

14. Alejandro Portes, "Migración y desarrollo: una revisión conceptual de la evidencia", op. cit. 
15. Francisco Alba, "Migración internacional y políticas públicas", op. cit.; Alejandro Portes, "Migración y desarrollo: una revisión conceptual de la evidencia", op. cit.

16. Jeffrey S. Passel, "Flujos migratorios México-Estados Unidos de 1990 a 2010: Un análisis preliminar basado en las fuentes de información estadounidenses", op. cit.

17. Voir tableau 1 et aussi René Zenteno, "Recent trends in mexican migration to US: The Mexico perspective", exposé présenté au 2011 Annual Meeting de la Population Association of America, Washington, 2 avril 2011. http://www.somede.org/documentos/zenteno-2011.pdf.; Jeffrey S. Passel, "Flujos migratorios México-Estados Unidos de 1990 a 2010: Un análisis preliminar basado en las fuentes de información estadounidenses", op. cit.

18. Silvia E. Giorguli et Édith Y. Gutiérrez, "Niños y jóvenes en el contexto de la migración internacional entre México y Estados Unidos", in Coyuntura Demográfica, n 1, 2011, pp. 21-25.

19. Jeffrey S. Passel, "Flujos migratorios México-Estados Unidos de 1990 a 2010: Un análisis preliminar basado en las fuentes de información estadounidenses", op. cit.

20. Francisco Alba, "Hacer virtud de la necesidad. Hacia una nueva generación de políticas para la migración México-Estados Unidos", in Este País. Tendencias y opiniones, n 246, 2011, pp. 8-12.

21. Ibid., p. 10.

22. Brígida García et Édith Pacheco, "La participación económica en el censo de población 2010", in Coyuntura Demográfica, $\mathrm{n}^{\circ} 1,2011$, pp. 35-38.

23. Stephen Castles, "Development and migration - Migration and development: What comes first?", op. cit.; Francisco Alba, "Migración internacional y políticas públicas", op. cit.

24. Silvia E. Giorguli et Édith Y. Gutiérrez, "Niños y jóvenes en el contexto de la migración internacional entre México y Estados Unidos", op. cit.

25. Silvia E. Giorguli et Paula Leite, "La integración socioeconómica de los mexicanos en Estados Unidos, 1980-2005: experiencia y prospectiva", in Francisco Alba, Manuel A. Castillo et Gustavo Verduzco (dir.), Los grandes problemas de México. Migraciones internacionales, vol. III, México, Le Collège de Mexique, 2010, pp. 355-394.

26. Francisco Alba, "Migración internacional y políticas públicas", op. cit.; Alejandro Portes, "Migración y desarrollo: una revisión conceptual de la evidencia", op. cit.

27. Francisco Alba, "Hacer virtud de la necesidad. Hacia una nueva generación de políticas para la migración México-Estados Unidos", op. cit.
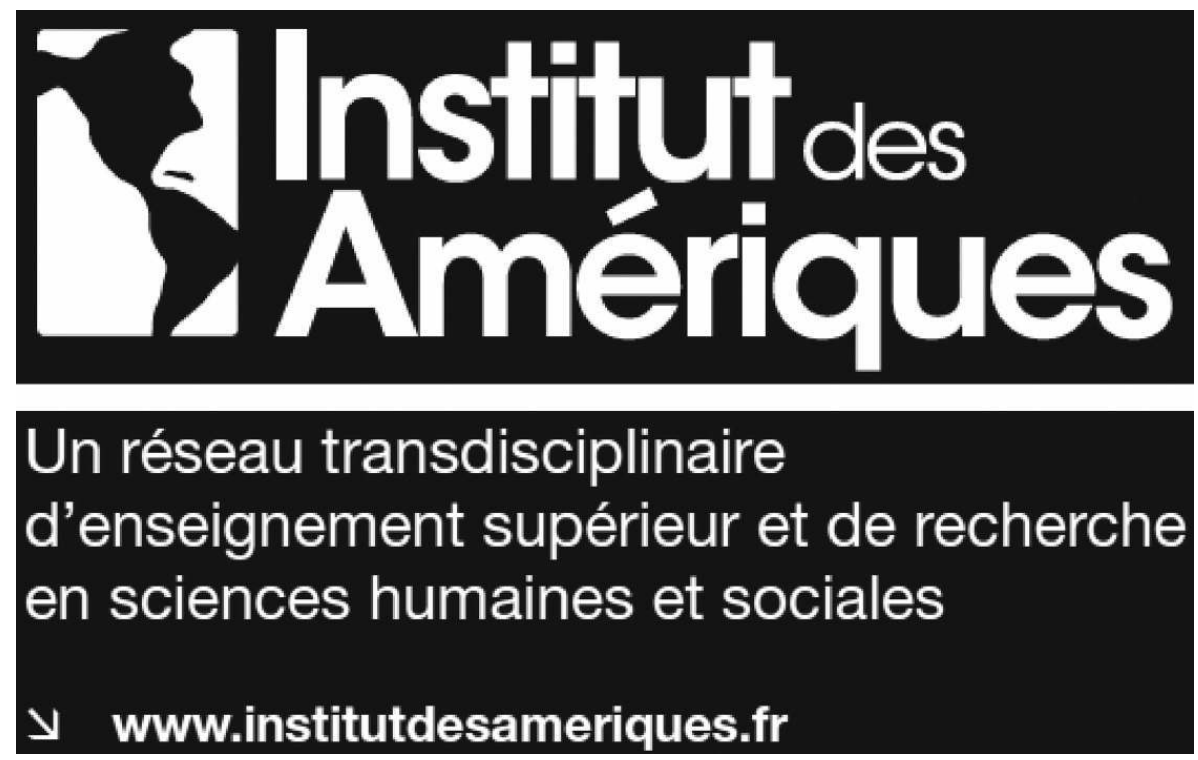